\title{
Diferencias en contenidos y situaciones de fantasías sexuales entre estudiantes de psicología de ambos sexos
}

José Moral de la Rubia*

Doctor en Filosofía y Ciencias de la Educación, Universidad de Alcalá de Henares, España. Profesor-investigador, Facultad de Psicología, Universidad Autónoma de Nuevo León, México. Correo electrónico: jose_moral@hotmail.com

Recibido: 19 de mayo del 2015 Aprobado: 1 de julio del 2015

Cómo citar este artículo: Diferencias en contenidos y situaciones de fantasías sexuales entre estudiantes de psicología de ambos sexos. Pensando Psicología, 11(18), 25-43. doi: http://dx.doi.org/10.16925/ pe.v11i18.1002

\section{Resumen}

Introducción: en México, se ha desarrollado el Cuestionario de Fantasías y Situaciones Sexuales (CFSs). Objetivos: describir sus distribuciones, estudiar diferencias entre sexos y proponer puntos de corte en estudiantes de psicología como futuros profesionales implicados en la atención a la salud sexual. Metodología: se empleó un muestreo por cuotas no proporcionales de sexo. Se aplicó el CFss a 400 estudiantes de psicología (200 mujeres y 200 hombres). Resultados: las distribuciones de los ítems, de las escalas de contenidos y situaciones $\mathrm{y}$ de los dos factores de contenidos mostraron sesgo hacia las frecuencias bajas. Los promedios en los ítems, en las dos escalas y en los dos factores de contenidos fueron mayores en hombres que en mujeres, salvo en fantasías de sexo con personas del mismo sexo, con personas maduras y con parejas concurrentes, así como en las situaciones antes o después de salir con la pareja y antes o después de dormir. Conclusiones: se concluye que debe realizarse una baremación por cuartiles diferenciales por sexo. Se sugiere el estudio y el empleo del cuestionario a fin de evaluar posibles sesgos que puedan afectar el juicio clínico entre los estudiantes de psicología.

Palabras clave: estudiantes de psicología, fantasías, México, psicometría, sexualidad. 


\title{
Differences in Contents and Situations of Sexual Fantasies among Psychology Students of Both Sexes
}

\begin{abstract}
Introduction: In Mexico, a Sexual Fantasies and Situations Questionnaire (CFSs) has been developed. Objectives: To describe distributions, study differences between sexes and propose cut-off points among psychology students as future professionals involved in providing sexual health attention. Methodology: A sample using non-proportional sex quotas was employed. The cFss was applied to 400 psychology students (200 women and $200 \mathrm{men}$ ). Results: The distributions of the items, of the scales of contents and situations and of the two content factors showed a bias towards low frequencies. The averages in the items, in the two scales and in the two content factors, were greater among men than among women, except for sexual fantasies with people of the same sex, with older people and with concurrent couples, as well as in situations before or after going out with a couple and before or after sleeping. Conclusions: It was concluded that a classification by sex differentiated quartiles must be made. The study and use of the questionnaire is suggested in order to avoid possible biases that could affect clinical judgment among psychology students.
\end{abstract}

Keywords: psychology students, fantasies, Mexico, psychometry, sexuality.

\section{Diferenças em conteúdos e situações de fantasias sexuais entre estudantes de psicologia de ambos os sexos}

\section{Resumo}

Introdução: no México, desenvolveu-se o Questionário de Fantasias e Situações Sexuais (QFSS). Objetivos: descrever suas distribuições, estudar diferenças entre sexos e propor pontos de corte em estudantes de psicologia como futuros profissionais implicados na atenção à saúde sexual. Metodologia: empregou-se uma amostra por cotas não proporcionais de sexo. O QFss foi aplicado a 400 estudantes de psicologia (200 mulheres e 200 homens). Resultados: as distribuições dos itens, das escalas de conteúdos e situações, e dos dois fatores de conteúdo mostraram uma tendência às frequências baixas. As médias nos itens, nas duas escalas e nos dois fatores de conteúdo, foram maiores em homens do que em mulheres, salvo em fantasias de sexo com pessoas do mesmo sexo, com pessoas maduras e com parceiros casuais, bem como nas situações antes ou depois de sair com o(a) parceiro(a) e antes ou depois de dormir. Conclusões: conclui-se que deve ser realizada uma avaliação por quartis diferenciais por sexo. Sugerem-se o estudo e o emprego do questionário a fim de avaliar possíveis tendências que possam afetar o julgamento clínico entre os estudantes de psicologia.

Palavras-chave: estudantes de psicologia, fantasias, México, psicometria, sexualidade. 


\section{Introducción}

La fantasía sexual es una cognición o conducta encubierta que implica imágenes y/o narrativas verbales excitadoras o estimulantes (Bartels y Gannon, 2011). Las fantasías sexuales pueden formar parte de una historia elaborada o representar actividades sexuales, cuerpos desnudos o áreas erógenas; pueden involucrar situaciones irreales, situaciones deseadas o construirse a partir de recuerdos e involucrar memorias episódicas. Se incluyen dentro de este concepto tanto las que ocurren espontáneamente como las que se producen intencionalmente (Birnbaum, 2007). Las fantasías sexuales también pueden ser compartidas. A veces son usadas por las parejas en la fase de excitación al mantener relaciones sexuales (Rathus, Nevid, Fichner-Rathus, Herold y McKenzie, 2005). Asimismo, los chismes de contenido sexual se pueden considerar fantasías sexuales grupales (Foster, 2004).

Entre las fantasías más frecuentes en adultos de ambos sexos, se encuentran revivir una experiencia sexual excitante e imaginar mantener relaciones sexuales con la pareja actual o con una pareja concurrente o nueva (Rathus et al., 2005). Entre las diferencias entre ambos sexos, cabe destacar que los hombres reportan mayor frecuencia de fantasías sexuales, contenidos sexuales más explícitos, mayor frecuencia de fantasías sobre tríos, orgías, encuentros casuales y dominación sexual. De igual forma, menor referencia a emociones y vínculo cuando comunican sus fantasías, y menor frecuencia de fantasías románticas y de sometimiento sexual (Carlstedt, Bood y Norlander, 2011; TuvalMashiach, Walsh, Harel y Shulman, 2008). Entre las diferencias también se halla que las mujeres sienten más culpa y celos que los hombres al fantasear con parejas concurrentes (Ahrold, Farmer, Trapnell y Meston, 2011). Los hombres tienen una mayor conciencia de excitación sexual durante las fantasías sexuales (Goldey, Avery y Van-Anders, 2014), las cuales van acompañadas de actividad sexual, como la masturbación, con más frecuencia en el hombre que en la mujer (Tuval-Mashiach et al., 2008). A estas diferencias unos investigadores les dan un origen filogenético (Buss, 2007; Schmitt et al., 2012), y otros un origen socio-cultural (Brotto, Jane, Woo y Gorzalka, 2012), si bien ambas perspectivas más que excluyentes son complementarias, ya que las estrategias fueron evolutivamente seleccionadas en contextos culturales (Wilson, 2010).
Las fantasías sexuales proveen una ventana a través de la cual se puede acceder a los deseos, objetivos y preferencias de los individuos (Birnbaum, 2007). De ahí la importancia que se concede a su estudio, especialmente en el ámbito clínico y forense (Williams, Cooper, Howell, Yuille y Paulhus, 2009). Debe señalarse que gran parte de la investigación en fantasías sexuales se ha realizado en población de estudiantes universitarios, especialmente en estudios con un enfoque diferencial entre sexos y culturas (Petersen y Hyde, 2010). Una de las justificaciones para el empleo de este tipo de muestras es la mayor sinceridad y actitud de colaboración de los estudiantes frente a otras poblaciones en temas de sexualidad y otros tópicos sensibles desde perspectivas éticas (De-Craene y Loopmans, 2014; Tourangeau y Yan, 2007), aunque la razón más importante es conocer los prejuicios, las representaciones y los conflictos de los futuros profesionales implicados en el tratamiento de problemas de pareja, disfunciones sexuales y parafilias (Leiblum, 2014; Maciel y Laganà, 2014).

Moral (2010) realizó un estudio sobre el contenido de las fantasías sexuales más frecuentes o dominantes en estudiantes mexicanos de psicología. Tras aplicarse un análisis de contenido, se obtuvo que el $52 \%$ de los estudiantes reportaron tener fantasías románticas o de sexo convencional con el novio o con la novia; el 19\% sobre sexo no convencional en actividad o lugar (actividades como sexo oral o coito anal exclusivas, o bien como elemento excitante central, así como mantener relaciones sexuales en lugares inusitados, siempre que se señale el lugar inusual como elemento más excitante y que no existan testigos que se excitan o sorprendan); el $7 \%$ varios contenidos con ninguno predominante; el 7\% sobre parejas concurrentes y orgías; el 5\% sobre sexo con famosos; el $3 \%$ sobre sometimiento sexual; el 3\% sobre sexo con casados/as, personas de distinta edad y familiares; y el $2 \%$ sobre exhibicionismo o voyerismo. Estas ocho clases de fantasías sexuales implicaban excluir actividad sexual entre personas del mismo sexo. En el 2\% restante aparecieron contenidos homosexuales o bisexuales. Se halló diferencia estadísticamente significativa en los contenidos de las fantasías entre ambos sexos $\left(\chi^{2}[8, N=209]=36,39, p<0,001\right)$. Las diferencias se marcaban en fantasías románticas ( $31 \%$ en hombres versus $59 \%$ en mujeres), de parejas concurrentes y orgías (19\% en hombres versus 3\% en mujeres), con casados/as, personas de distinta edad o familiares ( $10 \%$ en hombres versus $1 \%$ en mujeres), varias con ninguna en particular ( $4 \%$ en hombres versus $8 \%$ en mujeres), y con personas del mismo sexo 
( $0 \%$ en hombres versus $3 \%$ en mujeres). Asimismo, Moral (2010) encontró que las situaciones en las que estos estudiantes reportaron tener fantasías sexuales con más frecuencia fueron las de frustración amorosa y tensión sexual, lo cual es compatible con la teoría de la realización de deseos a través de las fantasías (Critelli y Bivona, 2008; Fisher y Greenberg, 1996).

Los estudios sobre fantasías sexuales se suelen realizar con escalas, ya que proporcionan datos fáciles de interpretar y analizar, así como con propiedades de fiabilidad y validez superiores a otros métodos (Wilson, 2010). Entre este tipo de instrumentos cabe destacar el Cuestionario de Fantasías Sexuales (Sex Fantasy Questionnaire -SFQ-) de Wilson, creado en 1978 en población estadounidense. Se compone de 40 ítems y cuatro factores correlacionados con consistencia interna alta: sexo exploratorio (sexo en grupo, parejas múltiples, concurrentes o del mismo sexo); fantasías de intimidad sexual (besar, sexo oral, hacer el amor con la pareja); fantasías de sexo impersonal (voyerismo, fetichismo, usar objetos para estimulación sexual); y fantasías de sometimiento/sumisión (Wilson, 2010). Está ampliamente trabajado y estandarizado en población estadounidense y ha sido adaptado a población española por Sierra, Ortega y Zubeidat (2006).

En México, se desarrolló el Cuestionario de Fantasías y Situaciones Sexuales (CFss; Moral, 2015). El cuestionario partió de un estudio cualitativo para lograr especificidad cultural en la población de estudiantes mexicanos de psicología. Inicialmente se redactaron 18 ítems de contenidos y diez ítems de situaciones. Finalmente se seleccionaron catorce ítems de contenido y los diez ítems de situaciones. Se eliminaron dos ítems sobre fantasías románticas o intimidad con la pareja por baja consistencia interna, por lo cual el cuestionario parece evaluar fantasías explícitas y excitadoras. La escala de contenidos mostró una estructura de dos factores correlacionados que tuvieron una consistencia interna buena. Un factor fue sobre fantasías heterosexuales de exploración; el otro factor fue sobre fantasías homosexuales, de sexo sin coito vaginal y pasividad (voyerismo y sometimiento). El modelo de dos factores correlacionados tuvo buen ajuste a los datos en el análisis unigrupo, pero propiedades de invarianza entre ambos sexos algo pobres en el análisis multigrupo. En mujeres, el modelo fue unidimensional con los catorce ítems seleccionados. Con los 18 ítems iniciales entre las mujeres se definieron dos factores: un factor de fantasías no románticas entre las que se incluyen las de contenido lésbico, y un factor de fantasías románticas entre las que se incluyen romances con hombres maduros y novios de amigas. El primero con consistencia interna excelente y el segundo con consistencia interna aceptable. En hombres, se reproduce el modelo de la muestra conjunta con los catorce ítems seleccionados (Moral, 2015). Aunque se ha determinado la consistencia interna y la estructura factorial del cuestionario CFSs, se desconocen las distribuciones de sus dos escalas y factores.

Con el fin de estimular la aplicación y estudio del cuestionario CFss en la población de estudiantes de psicología mexicanos y ahondar en el conocimiento de estos futuros profesionales implicados en el cuidado de la salud sexual, los objetivos de esta investigación son:

1. Describir las distribuciones de los 24 ítems, las dos escalas y los dos factores de contenido del CFss.

2. Contrastar las diferencias de medias entre los dos factores de contenidos en la muestra conjunta y en las muestras de ambos sexos.

3. Comparar las medias en los ítems de contenidos y situaciones, en las dos escalas y en los dos factores entre mujeres y hombres.

4. Estimar puntos de corte para las dos escalas y los dos factores de contenido dentro de la muestra.

\section{Método}

Se realizó un estudio no experimental con un diseño ex post facto transversal.

\section{Participantes}

Se empleó un muestreo no probabilístico. Se recolectó una muestra por cuotas no proporcionales de sexo de 400 participantes (200 hombres y 200 mujeres). Los criterios de inclusión fueron: ser estudiante de la facultad de psicología y prestar el consentimiento informado. Como criterios de exclusión fueron: cuestionario incompleto o valoración por parte del encuestador de que se contestó sin la debida atención. Los dos criterios de exclusión se impusieron a fin de conseguir datos de calidad.

La aplicación fue realizada por cuatro asistentes de investigación, dos de sexo femenino y dos de sexo masculino, quienes eran alumnos de la licenciatura en psicología con entrenamiento en técnicas de muestreo y evaluación psicológica. Los participantes fueron contactados en los jardines de la facultad y contestaron el cuestionario en las bancas o en aulas vacías. Los encuestadores permanecían cerca de la 
persona encuestada para resolver dudas mientras respondía el cuestionario. Al recoger el cuestionario, se aseguraban de que todas las preguntas estuvieran respondidas; en caso de que hubiera alguna en blanco, se pedía amablemente que se completase. La recolección de los datos se realizó entre enero y agosto del 2014.

La edad de los 400 participantes varió de 18 a 24 años, con una media de 20,16 años $(D E=1,56)$, siendo las medias de edad estadísticamente equivalentes entre ambos sexos en la muestra conjunta $(t[398]=$ $-0,39, p=0.700)$. El semestre cursado de licenciatura varió de primero a décimo con una media de cuarto semestre $(D E=2,55)$, siendo las medias de semestre cursado estadísticamente equivalentes entre ambos sexos $(t[398]=-0,43, p=0,666)$. De los 400 participantes, $392(98 \%)$ dijeron estar solteros y $8(2 \%)$ casados, siendo la distribución del estado civil estadísticamente equivalente entre ambos sexos $\left(\chi^{2}[1\right.$, $\mathrm{N}=400]=0,13, p=0,721$ con la corrección por continuidad de Yates). Con respecto al lugar de residencia, 333 de los 400 estudiantes (83\%) vivían con sus padres o familiares; 32 (8\%) en una casa de asistencia; 27 (7\%) compartiendo alquiler con amigos; y 8 (2\%) con su pareja; de manera que las distribuciones de frecuencia fueron estadísticamente equivalentes entre ambos sexos $\left(\chi^{2}[3, \mathrm{~N}=400]=1,36, p=\right.$ $0,715)$. Con respecto al estatus socioeconómico autodefinido, 243 de los 400 estudiantes (61\%) se consideraron de estatus medio-medio; 97 (24\%) medio-bajo; 57 (14\%) medio-alto; y 3 (1\%) alto. Ninguno se definió de estatus socioeconómico bajo. La mediana del estatus socioeconómico autodefinido correspondió a medio-medio, con diferencia de tendencia central estadísticamente significativa entre ambos sexos $\left(\mathrm{z}_{U}=\right.$ $-2,28, p=0,023)$. El promedio del estatus socioeconómico autodefinido fue significativamente mayor en las mujeres (rango promedio $=212,06$ ), que en los hombres (rango promedio $=188,94$ ). Con respecto a la afiliación religiosa, 339 de los 400 estudiantes (85\%) señalaron ser de religión cristiana católica; 27 (7\%) de religión cristiana no católica; y 34 (8\%) ateos o sin religión. La distribución de frecuencias de la afiliación religiosa fue diferencial entre ambos $\operatorname{sexos}\left(\chi^{2}[2, \mathrm{~N}=\right.$ $400]=12,87, p=0,002)$. Los hombres se definieron más como ateos o sin religión $(13,5 \%)$ que las mujeres (3,5\%; véase tabla 1$)$.

\section{Instrumento}

El Cuestionario de Fantasías y Situaciones Sexuales (CFss; Moral, 2015) fue desarrollado a raíz de un estudio cualitativo (Moral, 2010). Está compuesto por dos escalas. Una escala está integrada por catorce ítems que evalúan la frecuencia con la que se tienen ciertos contenidos de fantasías sexuales (por ejemplo, "hacer el amor con mi novio/a en un lugar romántico"). La otra escala está compuesta por diez ítems que evalúan la frecuencia con la que se tienen fantasías en situaciones específicas (por ejemplo, "después o antes de salir con mi pareja"). El formato de respuesta de los ítems de ambas escalas es de cinco categorías ordinales: 1 = "nunca", 2 = "muy rara vez", 3 = "a veces", 4 = "con frecuencia" y $5=$ "con mucha frecuencia" (véase Apéndice A).

Los 24 ítems del cuestionario son directos. La puntuación de cada escala se obtiene por la suma simple de ítems. La suma de los catorce primeros ítems del cuestionario proporciona la puntuación en la escala de contenidos y la suma de los diez últimos proporciona la puntuación en la escala de situaciones.

La estructura de la escala de contenidos fue de dos factores correlacionados. El primer factor quedó configurado por nueve indicadores (ítems $2,5,6,7,8$, $9,10,11$ y 12 ), tuvo consistencia interna buena ( $\alpha$ ordinal $=0,89$ ), y hace referencia a fantasías heterosexuales de exploración de nuevas experiencias (por ejemplo, "tener mi primera experiencia sexual"); con parejas concurrentes (por ejemplo, "ser infiel a mi pareja"); con parejas múltiples (por ejemplo, "participar en una orgía heterosexual"); y de distinta edad (por ejemplo, "hacerlo con una persona madura"). El segundo factor quedó conformado por cinco indicadores (ítems 1, 3, 4, 13 y 14), tuvo consistencia interna buena ( $\alpha$ ordinal $=0,89)$, $y$ hace referencia a fantasías de relaciones sexuales con personas del mismo sexo (por ejemplo, "tener relaciones homosexuales"); sexo sin coito vaginal ("ser masturbado/a, tener sexo oral o anal"); y pasividad sexual, incluyendo sometimiento ("qué me aten, me golpeen, me sometan o abusen de mí"); y voyerismo ("observar a otros teniendo relaciones sexuales"). Al contrastar el ajuste del modelo dos factores correlacionados por mínimos cuadrados sin ponderar, cinco de los seis índices de ajuste contemplados fueron buenos $(\chi 2 / g 1=1,87, G F I=0,97, A G F I$ $=0,96, N F I=0,96$ y $R F I=0,95)$, y uno fue adecuado $(S R M R=0,08)$. La correlación entre ambos factores fue significativa, alta y positiva $(r=0,62, p<0,001)$. Desde el análisis multigrupo con modelos anidados en constricciones acumulativas, las propiedades de invarianza del modelo bifactorial entre ambos sexos fueron algo pobres. Las diferencias se marcaron en el modelo con constricciones en las varianzas-covarianzas 
Tabla 1

Distribución de las variables sociodemográficas

\begin{tabular}{|c|c|c|c|c|c|c|c|c|}
\hline \multirow{2}{*}{ Variable } & \multirow{2}{*}{ Valores } & \multicolumn{3}{|c|}{ Muestra conjunta } & \multicolumn{2}{|c|}{ Mujeres } & \multicolumn{2}{|c|}{ Hombres } \\
\hline & & $\mathrm{f}$ & $\%$ & $\%$ acumulado & $\mathrm{f}$ & $\%$ & $\mathrm{f}$ & $\%$ \\
\hline \multirow{3}{*}{ Sexo } & Mujeres & 200 & 50 & & & & & \\
\hline & Hombres & 200 & 50 & & & & & \\
\hline & Total & 400 & 100 & & & & & \\
\hline \multirow{8}{*}{$\begin{array}{l}\text { Años } \\
\text { de edad }\end{array}$} & 18 & 70 & 17,5 & 17,5 & 37 & 18,5 & 33 & 16,5 \\
\hline & 19 & 80 & 20 & 37,5 & 42 & 21 & 38 & 19 \\
\hline & 20 & 90 & 22,5 & 60 & 40 & 20 & 50 & 25 \\
\hline & 21 & 78 & 19,5 & 79,5 & 39 & 19,5 & 39 & 19,5 \\
\hline & 22 & 47 & 11,75 & 91,25 & 27 & 13,5 & 20 & 10 \\
\hline & 23 & 30 & 7,5 & 98,75 & 12 & 6 & 18 & 9 \\
\hline & 24 & 5 & 1,25 & 100 & 3 & 1,5 & 2 & 1 \\
\hline & Total & 400 & 100 & & 200 & 100 & 200 & 100 \\
\hline \multirow{11}{*}{$\begin{array}{l}\text { Semestre } \\
\text { cursado }\end{array}$} & Primero & 75 & 18,75 & 18,75 & 39 & 19,5 & 36 & 18 \\
\hline & Segundo & 44 & 11 & 29,75 & 28 & 14 & 16 & 8 \\
\hline & Tercero & 54 & 13,5 & 43,25 & 21 & 10,5 & 33 & 16,5 \\
\hline & Cuarto & 52 & 13 & 56,25 & 26 & 13 & 26 & 13 \\
\hline & Quinto & 50 & 12,5 & 68,75 & 24 & 12 & 26 & 13 \\
\hline & Sexto & 41 & 10,25 & 79 & 20 & 10 & 21 & 10,5 \\
\hline & Séptimo & 34 & 8,5 & 87,5 & 16 & 8 & 18 & 9 \\
\hline & Octavo & 20 & 5 & 92,5 & 11 & 5,5 & 9 & 4,5 \\
\hline & Noveno & 19 & 4,75 & 97,25 & 9 & 4,5 & 10 & 5 \\
\hline & Décimo & 11 & 2,75 & 100 & 6 & 3 & 5 & 2,5 \\
\hline & Total & 400 & 100 & & 200 & 100 & 200 & 100 \\
\hline \multirow{3}{*}{ Estado civil } & Soltero/a & 392 & 98 & & 195 & 97,5 & 197 & 98,5 \\
\hline & Casado/a & 8 & 2 & & 5 & 2,5 & 3 & 1,5 \\
\hline & Total & 400 & 100 & & 200 & 100 & 200 & 100 \\
\hline \multirow{5}{*}{$\begin{array}{l}\text { Lugar de } \\
\text { residencia }\end{array}$} & Con sus padres & 333 & 83,25 & & 165 & 82,5 & 168 & 84 \\
\hline & Casa de asistencia & 32 & 8 & & 18 & 9 & 14 & 7 \\
\hline & Alquiler con amigos & 27 & 6,75 & & 12 & 6 & 15 & 7,5 \\
\hline & Con su pareja & 8 & 2 & & 5 & 2,5 & 3 & 1,5 \\
\hline & Total & 400 & 100 & & 200 & 100 & 200 & 100 \\
\hline \multirow{6}{*}{$\begin{array}{l}\text { Estatus socio- } \\
\text { económico } \\
\text { autodefinido }\end{array}$} & Bajo & 0 & 0 & 0 & 0 & 0 & 0 & 0 \\
\hline & Medio-bajo & 243 & 60,75 & 60,75 & 110 & 55 & 133 & 66,5 \\
\hline & Medio-medio & 97 & 24,25 & 85 & 48 & 24 & 49 & 24,5 \\
\hline & Media-alto & 57 & 14,25 & 99,25 & 39 & 19,5 & 18 & 9 \\
\hline & Alto & 3 & 0,75 & 100 & 3 & 1,5 & 0 & 0 \\
\hline & Total & 400 & 100 & & 200 & 100 & 200 & 100 \\
\hline \multirow{4}{*}{$\begin{array}{l}\text { Afiliación } \\
\text { religiosa }\end{array}$} & Cristiana católica & 339 & 84,75 & & 179 & 89,5 & 160 & 80 \\
\hline & Cristiana no católica & 27 & 6,75 & & 14 & 7 & 13 & 6,5 \\
\hline & Sin religión & 34 & 8,5 & & 7 & 3,5 & 27 & 13,5 \\
\hline & Total & 400 & 100 & & 200 & 100 & 200 & 100 \\
\hline
\end{tabular}

Nota. Elaboración propia. 
estructurales y en los residuos de medida. En mujeres el modelo resulta unidimensional por la alta correlación entre ambos factores $(r=0,834$ en el modelo sin constricciones). La separación de ambos factores es más atribuible a los hombres al ser la correlación entre los factores moderada-baja ( $r=0,393$ en el modelo sin constricciones; Moral, 2015).

La estructura de la escala de situaciones fue unifactorial. La consistencia interna de sus 10 ítems fue excelente ( $\alpha$ ordinal $=0,91$ ). Al contrastar el ajuste del modelo de un factor por mínimos cuadrados sin ponderar, cinco de los seis índices de ajuste contemplados fueron buenos $(\chi 2 / g l=1,51, G F I=0,98, A G F I=0,98, N F I$ $=0,98$ y $R F I=0,97)$, y uno fue adecuado $(S R M R=0,07)$. Desde el análisis multigrupo con modelos anidados en constricciones acumulativas, las propiedades de invarianza del modelo unifactorial entre ambos sexos fueron adecuadas (Moral, 2015).

\section{Procedimiento}

Paralaimplementación de estainvestigación se pidió el permiso del director de la facultad, quien prestó el apoyo para la realización del estudio. Se solicitó el consentimiento expreso informado de los participantes, se garantizó el anonimato de sus respuestas, no se solicitó ningún dato de identificación personal y se informó sobre la identidad del responsable del estudio, a quien se podía acudir para asesoría psicológica en relación con cualquier cuestión suscitada por el cuestionario. De este modo, se respetaron las normas éticas de investigación de la Sociedad Mexicana de Psicología (2007) y de la American Psychological Association (2002).

\section{Análisis de datos}

En relación con el primer objetivo de describir distribuciones, se usó la mediana como estadístico de tendencia central, el rango semi-intercuartílico como estadístico de variación y el coeficiente de asimetría intercuartílico de Yule-Bowley con los ítems. Las distribuciones de las dos escalas y los dos factores de contenido se describieron usando la media como estadístico de tendencia central, la desviación estándar como estadístico de variación, los deciles y los coeficientes de asimetría y curtosis de Fisher. Se contrastó el ajuste de las distribuciones de las dos escalas y los dos factores a una curva normal por la prueba de Shapiro-Paris.
En relación con el segundo objetivo de comparar las medias entre los dos factores de contenido en la muestra conjunta, en la de mujeres y en la de hombres, las diferencias se contrastaron por la prueba $t$ de Student para muestras emparejadas y el tamaño del efecto se estimó por la $d$ de Cohen (media de las puntuaciones diferenciales/desviación estándar de las puntuaciones diferenciales). Un valor de $d<0,2$ se interpretó como un tamaño de efecto trivial, entre 0,2 y 0,49 pequeño, entre 0,5 y 0,79 mediano y $\geq$ 0,8 grande. No obstante, de forma complementaria, la diferencia entre los dos factores de contenido se contrastó por la prueba de rangos asignados de Wilcoxon que no requiere variables con distribución normal.

En relación con el tercer objetivo de comparar medias entre ambos sexos, la diferencia de tendencia central de cada ítem se contrastó por la prueba $U$ de Mann-Whitney. Considerando la robustez de la prueba ante el incumplimiento de la normalidad cuando el rango de valores es continuo y el tamaño de muestra es grande, la diferencia de medias de cada escala y cada factor entre ambos sexos se contrastó por la prueba $t$ de Student para dos muestras independientes. La igualdad de varianza se contrastó por la prueba de Levene. En caso de heterocedasticidad o desigualdad de varianzas, se empleó la corrección de Welch-Satterthwaite. El tamaño del efecto se estimó por la $d$ de Cohen (diferencia de las medias/desviación estándar combinada). No obstante, de forma complementaria, estas diferencias se contrastaron por la prueba $U$ de Mann-Whitney que no requiere variables con distribución normal.

En relación con el cuarto objetivo de estimar puntos de corte dentro de la muestra, se emplearon los deciles para identificar los casos que se desvían por exceso $(\geq D 8)$ o defecto $(\leq D 2)$ de la tendencia central del conjunto de participantes, al no ajustarse a una curva normal las distribuciones de las dos escalas y los dos factores de contenido.

El nivel de significación se estipuló en 0,05. Los cálculos se realizaron con los programas SPSs21 y STATA12.

\section{Resultados}

\section{Distribuciones de los ítems, las escalas y los dos factores de contenido}

Los catorce ítems de contenido tuvieron un rango de 1 a 5, cubriendo así el rango potencial de valores. La mediana correspondió a 1 ("nunca") en once de los catorce contenidos (ítems 2, 3, 4, 5, 6, 7, 9, 11, 12, 13 
y 14), y correspondió a 2 ("muy rara vez") en tres contenidos (ítems 1, 8 y 10). Los ítems 3, 4 y 13 tuvieron un rango semi-intercuartílico nulo y un coeficiente de asimetría indeterminado, siendo la respuesta de la mayoría de los participantes "nunca" (entre 80,5 y $88 \%$ ). Las distribuciones de los ítems 2, 5, 6, 7, 9, 11, 12 y 14 mostraron asimetría positiva $(A I=1)$ o sesgo hacia valores de baja frecuencia, coincidiendo la mediana con el primer cuartil. La distribución del ítem 8 fue ligera asimetría positiva $(A I=0,33)$, quedando la mediana más próxima al primer cuartil que al tercero. Las distribuciones de los ítems 1 y 10 fueron simétricas $(A I=0)$, quedando la mediana en un valor intermedio entre el primer cuartil y el tercero (véase tabla 2).
Los diez ítems de situaciones también tuvieron un rango de 1 a 5 . La mediana correspondió a 1 ("nunca") en una de las diez situaciones (ítem 7), y correspondió a 2 ("muy rara vez") en las nueve situaciones restantes. El ítem 7 mostró asimetría positiva $(A I=1)$ o sesgo hacia valores bajos, coincidiendo la mediana con el primer cuartil. El ítem 4 mostró ligera asimetría positiva $(A I=0,33)$, quedando la mediana más próxima al primer cuartil que al tercero. Las distribuciones de los ocho restantes ítems fueron simétricas $(A I=0)$, quedando la mediana en un valor intermedio entre el primer cuartil y tercero (tabla 2).

A fin de obtener las puntuaciones de las dos escalas y dos factores de contenido en un rango

Tabla 2

Distribución y estadísticos descriptivos de los ítems de contenido y situaciones

\begin{tabular}{|c|c|c|c|c|c|c|c|c|c|c|}
\hline \multirow{2}{*}{ Ítems } & \multicolumn{5}{|c|}{ Frecuencia (\%) } & \multicolumn{5}{|c|}{ Descriptivos } \\
\hline & 1 & 2 & 3 & 4 & 5 & Q1 & Q2 & Q3 & RSI & AI \\
\hline \multicolumn{11}{|c|}{ Contenidos } \\
\hline c1 & $156(39 \%)$ & $64(16 \%)$ & $88(22 \%)$ & $62(15,5 \%)$ & $30(7,5 \%)$ & 1 & 2 & 3 & 1 & 0 \\
\hline c2 & $216(54 \%)$ & $70(17,5 \%)$ & $64(16 \%)$ & $30(7,5 \%)$ & $20(5 \%)$ & 1 & 1 & 3 & 1 & 1 \\
\hline c3 & $344(86 \%)$ & $16(4 \%)$ & $12(3 \%)$ & $14(3,5 \%)$ & $14(3,5 \%)$ & 1 & 1 & 1 & 0 & Ind \\
\hline c4 & $352(88 \%)$ & $6(1,5 \%)$ & $6(1,5 \%)$ & $14(3,5 \%)$ & $22(5,5 \%)$ & 1 & 1 & 1 & 0 & Ind \\
\hline c5 & $252(63 \%)$ & $72(18 \%)$ & $46(11,5 \%)$ & $16(4 \%)$ & $14(3,5)$ & 1 & 1 & 2 & 0,5 & 1 \\
\hline c6 & $280(70 \%)$ & $64(16 \%)$ & $24(6 \%)$ & $16(4 \%)$ & $16(4 \%)$ & 1 & 1 & 2 & 0,5 & 1 \\
\hline C7 & $298(74,5 \%)$ & $58(14,5 \%)$ & $20(5 \%)$ & $16(4 \%)$ & $8(2 \%)$ & 1 & 1 & 2 & 0,5 & 1 \\
\hline C8 & $142(35,5 \%)$ & $66(16,5 \%)$ & $82(20,5 \%)$ & $56(14 \%)$ & $54(13,5 \%)$ & 1 & 2 & 4 & 1,5 & 0,33 \\
\hline C9 & $248(62 \%)$ & $62(15,5 \%)$ & $50(12,5 \%)$ & $24(6 \%)$ & $16(4 \%)$ & 1 & 1 & 2 & 0,5 & 1 \\
\hline c10 & $152(38 \%)$ & $90(22,5 \%)$ & $84(21 \%)$ & $50(12,5 \%)$ & $24(6 \%)$ & 1 & 2 & 3 & 1 & 0 \\
\hline c11 & $280(70 \%)$ & $66(16,5 \%)$ & $30(7,5 \%)$ & $16(4 \%)$ & $8(2 \%)$ & 1 & 1 & 2 & 0,5 & 1 \\
\hline C12 & $222(55,5 \%)$ & $78(19,5 \%)$ & $60(15 \%)$ & $32(8 \%)$ & $8(2 \%)$ & 1 & 1 & 3 & 0,88 & 1 \\
\hline c13 & $322(80,5 \%)$ & $40(10 \%)$ & $16(4 \%)$ & $14(3,5 \%)$ & $8(2 \%)$ & 1 & 1 & 1 & 0 & Ind \\
\hline $\mathrm{C} 14$ & $300(75 \%)$ & $52(13 \%)$ & $30(7,5 \%)$ & $12(3 \%)$ & $6(1,5 \%)$ & 1 & 1 & 2 & 0,38 & 1 \\
\hline \multicolumn{11}{|c|}{ Situaciones } \\
\hline s1 & $164(41 \%)$ & $108(27 \%)$ & $90(22,5 \%)$ & $28(7 \%)$ & $10(2,5 \%)$ & 1 & 2 & 3 & 1 & 0 \\
\hline s2 & $102(25,5 \%)$ & $116(29 \%)$ & $100(25 \%)$ & $54(13,5 \%)$ & $28(7 \%)$ & 1 & 2 & 3 & 1 & 0 \\
\hline s3 & $164(41 \%)$ & $118(29,5 \%)$ & $86(21,5 \%)$ & $26(6,5 \%)$ & $6(1,5 \%)$ & 1 & 2 & 3 & 1 & 0 \\
\hline s4 & $142(35,5 \%)$ & $66(16,5 \%)$ & $84(21 \%)$ & $74(18,5 \%)$ & $34(8,5 \%)$ & 1 & 2 & 4 & 1,5 & 0,33 \\
\hline s5 & $118(29,5 \%)$ & $88(22 \%)$ & $122(30,5 \%)$ & $48(12 \%)$ & $24(6 \%)$ & 1 & 2 & 3 & 1 & 0 \\
\hline s6 & $184(46 \%)$ & $84(21 \%)$ & $66(16,5 \%)$ & $46(11,5 \%)$ & $20(5 \%)$ & 1 & 2 & 3 & 1 & 0 \\
\hline s7 & $260(65 \%)$ & $64(16 \%)$ & $50(12,5 \%)$ & $22(5,5 \%)$ & $4(1 \%)$ & 1 & 1 & 2 & 0,5 & 1 \\
\hline s8 & $182(45,5 \%)$ & $106(26,5 \%)$ & $60(15 \%)$ & $40(10 \%)$ & $12(3 \%)$ & 1 & 2 & 3 & 1 & 0 \\
\hline s9 & $172(43 \%)$ & $108(27 \%)$ & $86(21,5 \%)$ & $30(7,5 \%)$ & $4(1 \%)$ & 1 & 2 & 3 & 1 & 0 \\
\hline s10 & $168(42 \%)$ & $92(23 \%)$ & $76(19 \%)$ & $34(8,5 \%)$ & $30(7,5 \%)$ & 1 & 2 & 3 & 1 & 0 \\
\hline
\end{tabular}

Nota. $\mathrm{N}=400$. Cuartiles: $\mathrm{Q} 1, \mathrm{Q} 2=$ mediana y Q3. $\mathrm{RSI}=$ Rango semi-intercuartílico, AI = coeficiente de asimetría intercuartílico de Bowley. Ind = indeterminación al coincidir los tres cuartiles en el valor mínimo. Elaboración propia. 
homogéneo, se dividió la suma simple de ítems por el número de ítems sumados en cada escala o factor. Las cuatro distribuciones mostraron asimetría positiva $(A s>0)$. También presentaron apuntamiento $(C>0)$, salvo la distribución de la escala de situaciones que fue ligeramente aplanada. Así, ninguna se ajustó a una curva normal. Sus valores se concentraron en las puntuaciones bajas (véase la tabla 3). Al dividir el rango de posibles valores continuos $([1,5])$ en 5 intervalos de amplitud constante ([Max - Min]/ número de intervalos $=[5-1] / 5=0,8)$ para hacerlos corresponder con los cinco valores discretos de respuesta a cada ítem $([1 ; 1,8]=1=$ "nunca", $[1,81,2,6]$ $=2=$ "muy rara vez", $[2,61,3,4]=3=$ "a veces", $[3,1$, $4,2]=4=$ "con frecuencia" y $[4,21 ; 5]=5=$ "con mucha frecuencia"), la media de la escala de contenidos $(\mathrm{M}=1,74$; IC $95 \%: 1,67 ; 1,80)$, y la del factor de fantasías homosexuales, sexo sin coito vaginal y pasividad ( $\mathrm{M}=1,58$; IC $95 \%: 1,50 ; 1,65)$, correspondieron al intervalo $[1 ; 1,8]=1=$ "nunca". Más del $60 \%$ de las puntuaciones de ambas variables quedaron en dicho intervalo. La media del factor de fantasías heterosexuales de exploración $(M=1,83$, IC $95 \%$ : $1,76 ; 1,90)$ y la de escala de situaciones $(M=2,12$, IC $95 \%: 2,04 ; 2,20)$ correspondieron a $2=[1,81 ; 2,6]=$ “a veces" (véase tabla 3 ).

\section{Comparación de medias entre los dos factores de contenido}

Al comparar las medias por la prueba $t$ de Student para muestras emparejadas, la media en el factor de fantasías heterosexuales de exploración fue significativamente mayor que la del factor de fantasías homosexuales, de sexo sin coito vaginal y de pasividad ( $t$ $[399]=6,68, p<0,001)$, siendo la correlación entre ambos factores moderada $(r=0,49, p<0,001)$. Con una media de las puntuaciones diferenciales $=0,25$ y una desviación estándar de las puntuaciones diferenciales $=0,76$, el tamaño del efecto de la diferencia fue pequeño $(d$ de Cohen $=0,25 / 0,76=0,33$ ). La diferencia de medias también fue significativa al hacer la comparación por la prueba de Wilcoxon $(Z=-7,71$, $p<0,001)$

Tabla 3

Descriptivos en la muestra conjunta

\begin{tabular}{ccccc}
\hline Descriptivos & Contenidos & F1 & F2 & Situaciones \\
\hline$M[95 \% \text { IC }]^{*}$ & $1,74[1,67,1,80]$ & $1,83[1,76,1,90]$ & $1,58[1,50,1,65]$ & $2,12[2,04,2,20]$ \\
$D E$ & 0,66 & 0,75 & 0,77 & 0,81 \\
$A s[95 \% \text { IC }]^{*}$ & $1,41[1,17 ; 1,65]$ & $1,28[1,04 ; 1,52]$ & $1,95[1,71 ; 2,19]$ & $0,52[0,28 ; 0,76]$ \\
$C[95 \% \text { IC }]^{*}$ & $2,90[2,43 ; 3,38]$ & $1,66[1,19,2,14]$ & $3,89[3,41,4,37]$ & $-0,55[-1,03 ;-0,07]$ \\
$D 1[95 \% \text { IC }]^{* *}$ & $1[1 ; 1,07]$ & $1[1 ; 1]$ & $1[1 ; 1]$ & $1,10[1 ; 1,20]$ \\
$D 2[95 \% \text { IC }]^{* *}$ & $1,14[1,07 ; 1,21]$ & $1,13[1,11 ; 1,22]$ & $1[1 ; 1]$ & $1,30[1,20 ; 1,50]$ \\
$D 3[95 \% \text { IC }]^{* *}$ & $1,29[1,21 ; 1,36]$ & $1,33[1,22,1,44]$ & $1[1 ; 1]$ & $1,60[1,70 ; 1,90]$ \\
$D 4[95 \% \text { IC }]^{* *}$ & $1,43[1,36 ; 1,50]$ & $1,56[1,44 ; 1,56]$ & $1,20[1,20 ; 1,20]$ & $1,80[1,70 ; 1,90]$ \\
$D 5[95 \% \text { IC }]^{* *}$ & $1,64[1,56 ; 1,71]$ & $1,67[1,56 ; 1,78]$ & $1,40[1,20 ; 1,40]$ & $2,05[1,90 ; 2,20]$ \\
$D 6[95 \% \text { IC }]^{* *}$ & $1,79[1,71 ; 1,86]$ & $1,78[1,78,1,89]$ & $1,40[1,40 ; 1,60]$ & $2,20[2,20 ; 2,40]$ \\
$D 7[95 \% \text { IC }]^{* *}$ & $1,93[1,86 ; 2,02]$ & $2[2 ; 2,11]$ & $1,60[1,60 ; 1,80]$ & $2,50[2,40 ; 2,60]$ \\
$D 8[95 \% \text { IC }]^{* *}$ & $2,29[2,11 ; 2,29]$ & $2,33[2,22,2,44]$ & $2[1,80 ; 2,20]$ & $2,80[2,70 ; 3]$ \\
$D 9[95 \% \text { IC }]^{* *}$ & $2,57[2,45 ; 2,79]$ & $2,89[2,78 ; 3]$ & $2,60[2,40 ; 3]$ & $3,30[3,20 ; 3 ; 50]$ \\
$M a x$ & 4,86 & 4,89 & 4,80 & 4,20
\end{tabular}

Nota . Estadísticos: $\mathrm{M}=$ Media aritmética, $\mathrm{DE}=$ Desviación estándar, $\mathrm{A}=$ coeficiente de asimetría de Fisher, $\mathrm{C}=$ coeficiente de curtosis de Fisher y D $=$ deciles. ${ }^{*}$ Intervalo de confianza (IC) calculado bajo un modelo normal. ${ }^{* \star}$ Intervalo de confianza (IC) calculado bajo una interpolación binomial. zv' $=$ Prueba de normalidad de Shapiro-Paris. F1 = Fantasías heterosexuales de exploración $=\mathrm{C} 2+\mathrm{c} 5+\mathrm{c} 6+$ $\mathrm{C} 7+\mathrm{C} 8+\mathrm{C} 9+\mathrm{C} 10+\mathrm{C} 11+\mathrm{C} 12) \cdot \mathrm{F} 2=$ Fantasías homosexuales, de sexo sin coito vaginal y de pasividad $=\mathrm{C} 1+\mathrm{C} 3+\mathrm{C} 4+\mathrm{C} 13+\mathrm{C} 14$. Elaboración propia. 
En mujeres, la media en el factor de fantasías heterosexuales de exploración fue significativamente mayor que la del factor de fantasías homosexuales, de sexo sin coito vaginal y de pasividad $(t[399]=5,29$, $p<0,001)$, siendo la correlación entre ambos factores alta $(r=0,68, p<0,001)$. Con una media de las puntuaciones diferenciales $=0,30$ y una desviación estándar de las puntuaciones diferenciales $=0,92$, el tamaño del efecto de la diferencia fue pequeño $(d$ de Cohen $=0,30 / 0,92=0,33$ ). La diferencia de medias también fue significativa al hacer la comparación por la prueba de Wilcoxon $(Z=-6,67, p<0,001)$.

En hombres, la media en el factor de fantasías heterosexuales de exploración fue significativamente mayor que la del factor de fantasías homosexuales, de sexo sin coito vaginal y de pasividad $(t[399]$ $=4,62, p<0,001)$, siendo la correlación entre ambos factores moderada $(r=0,32, p<0,001)$. Con una media de las puntuaciones diferenciales $=0,21$ y una desviación estándar de las puntuaciones diferenciales $=0,55$, el tamaño del efecto de la diferencia fue pequeño $(d$ de Cohen $=0,21 / 0,55=0,38$ ).
La diferencia de medias también fue significativa al hacer la comparación por la prueba de Wilcoxon $(z=-4,92, p<0,001)$.

\section{Comparación de medias en los factores y las escalas entre ambos sexos}

Los promedios de los ítems de contenidos fueron significativamente más altos en los participantes masculinos que en las participantes femeninas, salvo en los contenidos de "hacer el amor con mi novio/a en un lugar romántico", "tener relaciones bisexuales", "tener relaciones homosexuales", "ser infiel a mi pareja" y "hacerlo con una persona madura", los cuales fueron estadísticamente equivalentes entre ambos sexos. También los promedios de los ítems de situaciones fueron significativamente más altos en los participantes masculinos que en las participantes femeninas, salvo en la situación de fantasear antes de dormir o al despertar, y en la situación después o antes de salir con mi pareja, las cuales fueron estadísticamente equivalentes entre ambos sexos (tabla 4).

Tabla 4

Comparación de tendencia central entre ambos sexos por la prueba $u$ de Mann-Whitney

\begin{tabular}{|c|c|c|c|c|}
\hline \multirow{2}{*}{ Ítems } & \multicolumn{2}{|c|}{ Rango promedio } & \multicolumn{2}{|c|}{ Mann-Whitney } \\
\hline & Mujer & Hombre & Zu & $\mathrm{p}$ \\
\hline \multicolumn{5}{|l|}{ Contenidos excluidos } \\
\hline Hacer el amor con mi novio/a en un lugar romántico & 191,13 & 209,87 & $-1,68$ & 0,093 \\
\hline Tener mi primera experiencia sexual & 183,05 & 217,95 & $-3,12$ & 0,002 \\
\hline Hacerlo con un primo o alguien de la familia & 190,96 & 210,04 & $-2,53$ & 0,012 \\
\hline $\begin{array}{l}\text { Hacerlo en un sitio donde haya peligro de que nos sorprendan o estén obser- } \\
\text { vando }\end{array}$ & 164,10 & 236,90 & $-6,56$ & $<0,001$ \\
\hline \multicolumn{5}{|l|}{ Los 14 ítems de contenidos del CFss } \\
\hline c1. Ser masturbado/a, tener sexo oral o anal & 156,64 & 244,36 & $-7,90$ & $<0,001$ \\
\hline c2. Tener relaciones sexuales en un avión, manejando o en situaciones de riesgo & 182,27 & 218,73 & $-3,46$ & 0,001 \\
\hline c3. Tener relaciones bisexuales & 194,89 & 206,11 & $-1,61$ & 0,108 \\
\hline c4. Tener relaciones homosexuales & 196,30 & 204,70 & $-1,29$ & 0,198 \\
\hline c5. Ser infiel a mi pareja & 191,46 & 209,54 & $-1,82$ & 0,070 \\
\hline c6. Participar en una orgía heterosexual & 172,86 & 228,14 & $-5,92$ & $<0,001$ \\
\hline c7. Hacer un intercambio de parejas & 174,98 & 226,02 & $-5,78$ & $<0,001$ \\
\hline c8. Hacerlo con un hombre famoso o una mujer famosa & 181,51 & 219,49 & $-3,39$ & 0,001 \\
\hline C9. Ser el amante o hacerlo con una persona casada & 178,16 & 222,84 & $-4,45$ & $<0,001$ \\
\hline c10. Hacerlo con una persona madura & 190,67 & 210,33 & $-1,77$ & 0,077 \\
\hline c11. Tener relaciones sexuales con el/la novio/a de mi amigo/a & 173,12 & 227,88 & $-5,87$ & $<0,001$ \\
\hline
\end{tabular}


(viene)

\begin{tabular}{|c|c|c|c|c|}
\hline \multirow{2}{*}{ Ítems } & \multicolumn{2}{|c|}{ Rango promedio } & \multicolumn{2}{|c|}{ Mann-Whitney } \\
\hline & Mujer & Hombre & $\mathbf{z U}$ & p \\
\hline $\mathrm{c} 12$. Tener relaciones con alguien mucho más joven que yo & 165,31 & 235,69 & $-6,73$ & $<0,001$ \\
\hline c13. Qué me aten, me golpeen, me sometan o abusen de mí & 190,07 & 210,93 & $-2,61$ & 0,009 \\
\hline c14. Observar a otros teniendo relaciones sexuales & 187,73 & 213,27 & $-2,91$ & 0,004 \\
\hline \multicolumn{5}{|l|}{ Los 10 ítems de situaciones del CFss } \\
\hline s1. Cuando estudio o estoy aburrido & 177,85 & 223,15 & $-4,13$ & $<0,001$ \\
\hline s2. Antes de dormir o al despertar & 193,19 & 207,81 & $-1,30$ & 0,192 \\
\hline s3. Viendo la televisión & 173,91 & 227,09 & $-4,86$ & $<0,001$ \\
\hline s4. Cuando llevo muchos días sin masturbarme o tener actividad sexual & 163,13 & 237,87 & $-6,69$ & $<0,001$ \\
\hline s5. Después o antes de salir con mi pareja & 190,64 & 210,36 & $-1,77$ & 0,077 \\
\hline $\begin{array}{l}\text { s6. Cuando deseo a alguien que no me atrevo a conquistarlo/a o no me es } \\
\text { accesible }\end{array}$ & 176,77 & 224,23 & $-4,36$ & $<0,001$ \\
\hline s7. Cuando deseo a alguien que me rechaza & 171,51 & 229,49 & $-5,91$ & $<0,001$ \\
\hline s8. Cuando estoy en el chisme o se platica de cosas sexuales & 184,66 & 216,34 & $-2,92$ & 0,004 \\
\hline s9. Cuando estoy en la ducha o el baño & 166,05 & 234,95 & $-6,32$ & $<0,001$ \\
\hline s10. Después de tener actividad sexual con mi pareja & 175,84 & 225,16 & $-4,48$ & $<0,001$ \\
\hline \multicolumn{5}{|l|}{ Escalas y factores del cFss } \\
\hline Escala de contenidos & 159,03 & 241,97 & $-7,18$ & $<0,001$ \\
\hline Factor de fantasías heterosexuales de exploración & 166,42 & 234,58 & $-5,91$ & $<0,001$ \\
\hline Factor de fantasías homosexuales, de sexo sin coito vaginal y de pasividad & 160,89 & 240,11 & $-7,03$ & $<0,001$ \\
\hline Escala de situaciones & 166,66 & 234,34 & $-5,86$ & $<0,001$ \\
\hline
\end{tabular}

Nota. Elaboración propia.

Al comparar las medias por la prueba $t$ de Student para muestras independientes, las medias de los participantes masculinos fueron significativamente más altas en las dos escalas y en los dos factores de contenido que las medias de las participantes femeninas $(p<0,001)$. El tamaño del efecto del sexo fue mediano sobre las escalas de contenido ( $d$ de Cohen $=-0,64$ ); de situaciones $(d$ de Cohen $=-0,57)$; y sobre el factor de fantasías heterosexuales de exploración $(d$ de Cohen $=$ $-0,61$ ); también fue pequeño sobre el factor de fantasías homosexuales, de sexo sin coito vaginal y de pasividad ( $d$ de Cohen $=-0,46$; tabla 5 ). Estas diferencias fueron de igual modo significativas al hacer la comparación por la prueba $U$ de Mann-Whitney (tabla 4).

Tabla 5

Comparación de medias entre ambos sexos por la prueba t de Student

\begin{tabular}{ccccccccc}
\hline \multirow{2}{*}{ CFSs } & \multicolumn{9}{c}{ Medias [IC 95\%] } & \multicolumn{2}{c}{ Levene } & \multicolumn{2}{c}{ Student } & \multirow{2}{*}{$d$ de Cohen } \\
\cline { 2 - 7 } & Mujeres & Hombres & F & $p$ & $t$ & $g l$ & $p$ & \\
\hline Cont. & $1,54[1,45,1,62]$ & $1,94[1,85,2,03]$ & 4,25 & 0,040 & $-6,39$ & 394,65 & $<0,001$ & $-0,64[-0,84,-0,44]$ \\
F1 & $1,61[1,53,1,70]$ & $2,05[1,94,2,16]$ & 17,70 & $<0,001$ & $-6,09$ & 373,36 & $<0,001$ & $-0,61[-0,81,-0,41]$ \\
F2 & $1,40[1,30,1,50]$ & $1,75[1,64,1,85]$ & 3,47 & 0,063 & $-4,58$ & 398 & $<0,001$ & $-0,46[-0,66,-0,26]$ \\
Situa. & $1,90[1,79,2,01]$ & $2,34[2,23,2,45]$ & 0,01 & 0,935 & $-5,71$ & 398 & $<0,001$ & $-0,57[-0,77,-0,37]$ \\
\hline
\end{tabular}

Nota. $\mathrm{F} 1$ = Fantasías heterosexuales de exploración $=\mathrm{c} 2+\mathrm{c} 5+\mathrm{c} 6+\mathrm{c} 7+\mathrm{c} 8+\mathrm{c} 9+\mathrm{c} 10+\mathrm{c} 11+\mathrm{c} 12$. F2 = Fantasías homosexuales, de sexo sin coito vaginal y de pasividad $=\mathrm{c} 1+\mathrm{c} 3+\mathrm{c} 4+\mathrm{c} 13+\mathrm{c} 14$. Elaboración propia. 


\section{Estimación de puntos de corte para las dos escalas y los dos factores de contenido}

Debido a que las distribuciones no se ajustaron a una curva normal, su baremación debe realizarse por los deciles segundo y octavo. En la presente muestra no probabilística, las puntuaciones $\leq 1,14$ en la escala de contenidos, $\leq 1,13$ en el factor de fantasías heterosexuales de exploración, $\mathrm{y} \leq 1,30$ en la escala de situaciones, identificaron a los participantes con una desviación marcada por debajo de la tendencia central. El factor de fantasías homosexuales, de sexo sin coito vaginal y de pasividad no tuvo punto de corte inferior, ya que el valor 1 apareció en 140 de los 400 participantes (35\%). Las puntuaciones $\geq 2,29$ en la escala de contenidos, $\geq 2,33$ en el factor de fantasías heterosexuales de exploración, $\geq 2$ en el factor de fantasías homosexuales, de sexo sin coito vaginal $y$ de pasividad, $y \geq 2,80$ en la escala de situaciones identificaron a los participantes con una desviación marcada por encima de la tendencia central (tabla 6). Al existir diferencia significativa entre ambos sexos, está justificado considerar baremos diferenciales entre los hombres y mujeres que participaron en el estudio.

Entre las participantes femeninas, las puntuaciones $\leq 1,07$ en la escala de contenidos, $\leq 1,11$ en el factor de fantasías heterosexuales de exploración, $y \leq 1,20$ en la escala de situaciones, identificaron a aquéllas con una desviación marcada por debajo de la tendencia central. Para el factor de fantasías homosexuales, de sexo sin coito vaginal y de pasividad, no hubo punto de corte, ya que el valor 1 apareció en 106 de las 200 mujeres (53\%). Las puntuaciones $\geq 1,86$ en la escala de contenidos, $\geq 2$ en el factor de fantasías heterosexuales de exploración, $\geq 1,60$ en el factor de fantasías homosexuales, de sexo sin coito vaginal y de pasividad, $y \geq 2,50$ en la escala de situaciones identificaron a aquellas con una desviación marcada por encima de la tendencia central. En mujeres los dos factores de contenidos podrían ser ignorados, ya que la escala es unidimensional (véase tabla 6).

Entre los participantes masculinos, las puntuaciones $\leq 1,36$ en la escala de contenidos, $\leq 1,36$ en el factor de fantasías heterosexuales de exploración, $\leq$ 1,20 en el factor de fantasías homosexuales, de sexo sin coito vaginal y de pasividad, $y \leq 1,70$ en la escala de situaciones identificaron a aquéllos con una desviación marcada por debajo de la tendencia central. Las puntuaciones $\geq 2,49$ en la escala de contenidos, $\geq$ 2,78 en el factor de fantasías heterosexuales de exploración, $\geq 2,36$ en el factor de fantasías homosexuales, de sexo sin coito vaginal y de pasividad, $y \geq 3,08$ en la escala de situaciones identificaron a aquéllos con una desviación marcada por encima de la tendencia central (tabla 6).

\section{Discusión}

En respuesta al primer objetivo, se describieron las distribuciones. En la presente muestra no probabilística de 400 estudiantes de psicología, la distribución

Tabla 6

Deciles en la muestra de mujeres y de hombres

\begin{tabular}{|c|c|c|c|c|c|c|c|c|}
\hline \multirow{2}{*}{ Deciles } & \multicolumn{4}{|c|}{ Mujeres } & \multicolumn{4}{|c|}{ Hombres } \\
\hline & Contenidos & F1 & F2 & Situaciones & Contenidos & F1 & F2 & Situaciones \\
\hline $\mathrm{D} 1$ & 1 & 1 & 1 & 1 & 1,15 & 1,11 & 1 & 1,31 \\
\hline $\mathrm{D} 2$ & 1,07 & 1,11 & 1 & 1,20 & 1,36 & 1,36 & 1,20 & 1,70 \\
\hline D3 & 1,14 & 1,22 & 1 & 1,30 & 1,50 & 1,56 & 1,26 & 1,90 \\
\hline $\mathrm{D} 4$ & 1,29 & 1,33 & 1 & 1,54 & 1,71 & 1,67 & 1,40 & 2,10 \\
\hline D5 & 1,36 & 1,50 & 1 & 1,70 & 1,79 & 1,78 & 1,40 & 2,30 \\
\hline D6 & 1,57 & 1,67 & 1,20 & 1,96 & 1,93 & 2 & 1,60 & 2,50 \\
\hline D7 & 1,69 & 1,78 & 1,40 & 2,20 & 2,26 & 2,33 & 1,80 & 2,70 \\
\hline $\mathrm{D} 8$ & 1,86 & 2 & 1,60 & 2,50 & 2,49 & 2,78 & 2,36 & 3,08 \\
\hline D9 & 2,29 & 2,33 & 2,20 & 3,19 & 2,85 & 3,31 & 2,98 & 3,58 \\
\hline
\end{tabular}

Nota. $\mathrm{F} 1$ = Fantasías heterosexuales de exploración $=\mathrm{c} 2+\mathrm{c} 5+\mathrm{c} 6+\mathrm{C} 7+\mathrm{c} 8+\mathrm{c} 9+\mathrm{c} 10+\mathrm{c} 11+\mathrm{c} 12$. F2 = Fantasías homosexuales, de sexo sin coito vaginal y de pasividad $=\mathrm{c} 1+\mathrm{c} 3+\mathrm{c} 4+\mathrm{c} 13+\mathrm{c} 14$. Elaboración propia. 
de la escala, de los dos factores y de los ítems de contenido, salvo los ítems c1 y c10, mostraron sesgo hacia los valores de baja frecuencia $(1=$ "nunca" y 2 = "muy rara vez"), con lo cual se desviaron de un modelo de curva normal. Aunque la mayoría de los ítems de situaciones mostraron simetría y los índices de simetría y curtosis de la escala de situaciones se aproximaron más a los valores nulos - característicos de la normalidad-, finalmente la distribución de esta escala tampoco se ajustó a una curva normal; de ahí la necesidad de emplear cuantiles para baremar las dos escalas y los dos factores en la presente muestra por cuotas de sexo no proporcionales a las cuotas poblacionales, en lugar de establecer los baremos con la media y la desviación estándar.

La distribución normal es propia de rasgos adaptativos y sometidos a selección natural, como la capacidad para el pensamiento abstracto, $y$ las distribuciones con asimetría negativa y apuntamiento son propias de rasgos desadaptativos, como las parafilias, o sometidos a desaprobación social, como la desviación de la heterosexualidad (Kaplan y Saccuzzo, 2009). ¿Por qué las frecuencias con que se reporta tener las fantasías sexuales y estar en las situaciones que se enlistan el cuestionario son bajas? ¿La tendencia a tener fantasías sexuales es un rasgo desadaptativo o sometido a desaprobación social?

El cuestionario inicialmente contó con dos contenidos sobre fantasías románticas y de intimidad con una pareja heterosexual, los cuales fueron los reportados con más frecuencia. Así, solo el 14\% de los participantes reportaron nunca haber fantaseado hacer el amor con su novio/a en un lugar romántico, y el 27\% nunca haber fantaseado tener su primera experiencia sexual, cuando el porcentaje de nunca varió de 36 a $88 \%$ con un promedio de $64 \%$ entre los catorce contenidos incluidos en la escala. No obstante, las dos fantasías románticas y de intimidad con la pareja mostraron problemas de consistencia interna entre las participantes femeninas y entre los participantes masculinos. La tendencia general a la baja frecuencia en el reporte de los contenidos, el mayor promedio de los participantes masculinos y la baja consistencia de las fantasías románticas parecen indicar que el cuestionario evalúa fantasías sexuales explícitas y excitadoras, de manera que los contenidos sutiles, velados o románticos resultan inconsistentes (Moral, 2015; Sylva et al., 2013).

Cabe preguntarse si la baja frecuencia de reporte corresponde a la realidad o si el contenido explícito sexual genera un sesgo atenuador mediado por la deseabilidad social. Al evaluar la persona encuestada que reporta la verdadera frecuencia de fantasías sexuales explícitas se podría interpretar como signo de patología o motivar un descrédito social. Varias investigaciones revelan que las fantasías románticas y de intimidad son las más frecuentes, más en mujeres que en hombres, pero seguidas de cerca de fantasías de sexo con una pareja concurrente y fantasías sexuales de exploración (Rathus et al., 2005; Wilson, 2010), las cuales corresponden al contenido de la mayoría de los ítems del CFss. Así, entre los 400 participantes del presente estudio, los tres contenidos reportados con más frecuencia fueron "ser masturbado/a, tener sexo oral o anal", "hacerlo con un hombre famoso o una mujer famosa" y "hacerlo con una persona madura". Los contenidos claramente parafílicos sí son menos frecuentes, muchos de los cuales están ausentes en el CFss, tales como sadismo o coerción sexual, pedofilia, travestismo, exhibicionismo y fetichismo. Precisamente, el único presente, el de sometimiento sexual, fue de las fantasías menos reportadas junto con las fantasías homosexuales y bisexuales. Debe considerarse que los ítems fueron redactados a partir de los temas presentes al detallar fantasías favoritas, frecuentes o salientes entre estudiantes de psicología.

En caso de existir un sesgo deflactor, este podría estar motivado por una representación de la sexualidad como potencialmente adictiva, la cual puede generar problemas o descrédito y busca ocultamiento y control. Precisamente, esta representación sí está presente en la cultura latina a la que pertenecen los participantes del estudio, como muestran estudios realizados en Uruguay (Darré, 2005), Argentina (Morgade, 2006), o México (Vázquez y Chávez, 2008). Asimismo, Moral y Ortega (2009) hallaron, en esta misma población de estudiantes de psicología, una representación de la sexualidad muy ligada a los mensajes de educación sexual y el establecimiento de una identidad heterosexual, esto es, a comunicar un estándar de control y normalidad. Considerando estos estudios, podría conjeturarse la existencia de un sesgo atenuador.

En respuesta al segundo objetivo se compararon los dos factores de contenidos dentro de cada participante. Los contenidos heterosexuales de exploración fueron reportados con más frecuencia que los contenidos homosexuales, de sexo sin coito vaginal y pasividad, como cabe esperar desde el heterosexismo dominante en la cultura occidental, a la que pertenecen los participantes del estudio (Herek y McLemore, 2013). Bajo la ideología heterosexista se 
valora la heterosexualidad como la única orientación sexual natural, sana y deseable para el funcionamiento correcto de la sociedad, y se desvaloriza y margina cualquier desviación de la misma (Morgade, 2006).

La diferencia entre los dos factores de contenido fue mayor en los participantes masculinos que en las participantes femeninas, lo cual es concordante con el hecho de que los factores estén claramente diferenciados en los participantes masculinos con un $10 \%$ de varianza compartida, pero su distinción sea más confusa en las participantes femeninas (Moral, 2015). Esta mayor diferenciación es comprensible desde unos valores homofóbicos más centrados en la sexualidad masculina que en la femenina, con una condena abierta de la conducta homosexual en hombres y una tolerancia hacia la conducta lésbica, sobre todo si es puesta al servicio de la excitación masculina a través de espectáculos, películas, tríos, intercambios de parejas y orgías (Herek y McLemore, 2013). No obstante, la frecuencia de reporte en ambos factores fue significativamente mayor en los participantes masculinos que en las participantes femeninas, lo que se podría explicar por la mayor tendencia de los hombres a reportar contenidos explícitos y excitación en sus fantasías sexuales con menor influencia de la deseabilidad social que las mujeres (Goldey et al., 2014; Huberman, Suschinsky, Lalumière y Chivers, 2013; Seifert, Boulas, Huss y Scalora, 2011). Esta tendencia se atribuye a una mayor libertad sexual que la sociedad occidental concede al hombre en relación con la que otorga a la mujer, salvo en la conducta homosexual (Brotto et al. 2012), y a un impulso sexual mayor en el hombre que en la mujer (Goldey et al., 2014; Schmitt et al., 2012). Si la frecuencia de masturbación, búsqueda de actividad sexual y los niveles de testosterona se consideran como indicadores del impulso sexual, estos indicadores no solo son más altos en los hombres en comparación con las mujeres en la especie humana, sino también en machos de diferentes especies en comparación con las hembras de su misma especie (Schmitt et al., 2012), por lo cual la diferencia tendría una base socio-biológica.

En respuesta al tercer objetivo se compararon las medias entre los participantes de ambos sexos. Los promedios en los ítems de contenido fueron más altos en los participantes masculinos que en las participantes femeninas, como se reporta en otros estudios cuando los contenidos se refieren a contenidos sexuales explícitos (Holly, 2005; Rathus et al., 2005; Wilson, 2010). Solo hubo equivalencia de promedios en cuatro contenidos de la escala relacionados con prácticas sexuales con personas del mismo sexo, tener sexo con una persona madura y tener sexo con una pareja concurrente. En el ítem excluido de hacer el amor con mi novio/a en un lugar romántico tampoco hubo diferencia entre los participantes de ambos sexos.

En comparación con las conductas sexuales con personas del mismo sexo que son poco frecuentes y con un porcentaje de casos mayor en hombres que en mujeres, las fantasías sexuales con personas del mismo sexo son más frecuentes y con un porcentaje de casos estadísticamente equivalente entre ambos sexos (Moral, 2010; Remafedi, Resnick, Blum y Harris, 1992), o con un porcentaje mayor en mujeres que en hombres (Binter et al., 2012; Vrangalova y Savin-Williams, 2010; Wilson, 2010). En la presente muestra no probabilística, las fantasías con contenidos bisexuales estuvieron presentes en el 14\% de los participantes (95\% IC: 10,6\%, 17,4\%); 17\% (95\% IC: $11,8 \%, 22,2 \%)$ de los participantes masculinos; y $11 \%$ (95\% IC: $6,6 \%, 15,4 \%$ ) de las participantes femeninas, sin que exista diferencia estadísticamente significativa entre ambos sexos $(p>0,05)$, como tampoco se observó en los estudios de Moral (2010) y Remafedi, Resnick, Blum y Harris (1992). Estos porcentajes son estadísticamente equivalentes a los reportados por Moral (2010) en estudiantes de psicología mexicanos, como muestran los solapamientos de las estimaciones con intervalos de confianza del 95\%: 20,9\% (95\% IC: $16,9 \%, 24,9 \%$ ) en la muestra conjunta; $22,4 \%$ (95\% IC: $12,3 \%, 32,5 \%$ ) en la muestra de hombres; y $20,7 \%$ (95\% IC: $16,3 \%, 25 \%$ ) en la muestra de mujeres (Moral, 2010).

Tener sexo con una persona madura fue uno de los contenidos más frecuentes en la presente muestra no probabilística. Aunque su frecuencia fue estadísticamente equivalente entre los participantes de ambos sexos, parece tener un significado distinto para las participantes femeninas que para los participantes masculinos. Al explorar la estructura factorial en la muestra de las 200 estudiantes femeninas con los 18 ítems iniciales, se definió un factor con consistencia interna aceptable que incluía entre las fantasías románticas el tener sexo con un hombre maduro y con el novio de una amiga. En la muestra de los 200 estudiantes masculinos, el ítem quedó entre las fantasías heterosexuales de exploración. Congruente con la perspectiva evolutiva, el tener un romance con un hombre maduro, atractivo y exitoso o tener un romance con el novio de una amiga, implica triunfo dentro de la competencia reproductiva intragénero, al ser elegida como el verdadero amor y objeto de 
deseo, y no como amante. En estos hombres jóvenes el tener sexo con mujeres maduras se relaciona con experimentar y explorar en el campo de la sexuali$\mathrm{dad}$, al igual que tener una pareja concurrente, hacer intercambio de parejas, participar en una orgía o ser amante de una mujer casada.

Otro contenido frecuente y sin diferencia de frecuencia entre los participantes de ambos sexos de la presente muestra no probabilística fue el de una pareja concurrente, como también aparece en otros estudios (Rathus et al., 2005; Wilson, 2010). Los valores de fidelidad y exclusividad del romanticismo podrían implicar una correlación significativa y negativa entre tener fantasías románticas y de infidelidad, y una saturación negativa y mayor que 0,30 del ítem de infidelidad en el factor de fantasías románticas. No obstante, cuando se exploró la estructura factorial de los 18 ítems originales de la escala, se observó que el contenido de tener una pareja concurrente fue independiente del factor de fantasías románticas, tanto en la muestra conjunta como en la de hombres, y en la de mujeres; las cargas del ítem fueron positivas y menores a 0,10 en el factor de fantasías románticas en las tres muestras. Asimismo, la correlación entre los contenidos de intimidad e infidelidad con la pareja fue positiva tanto en la muestra conjunta $\left(r_{s}=0,09, p\right.$ $=0,06$; correlación policórica $[P C C]=0,13, p<0,01$ ), como en la de mujeres $\left(r_{s}=0,13, p=0,07, P C C=0,19\right.$; $p<0,01)$, y en la de hombres $\left(r_{S}=0,04, p=0,52, P C C\right.$ $=0,08, p=0,26)$. Esta correlación positiva no fue significativa en ninguna de las tres muestras al ser estimada por el coeficiente de correlación de rangos ordenados de Spearman, pero sí fue significativa en la muestra conjunta y en la de mujeres al ser estimada por el coeficiente de correlación policórica, el cual se considera que proporciona como estimación más exacta de la verdadera relación entre dos variables ordinales (Zumbo, Gadermann y Zeisser, 2007). Por lo tanto, tener más fantasías románticas con la pareja no implicó tener menos fantasías de serle infiel entre los participantes de este estudio, sino que ambos contenidos resultaron independientes entre los participantes masculinos y mostraron una tendencia muy débil a coexistir entre las participantes femeninas. Debe señalarse que las fantasías sobre parejas concurrentes se incrementan con los años de relación, tal como lo reflejan otros estudios (Birnbaum, 2007; Brotto et al., 2012). De ahí que, en otras poblaciones - como en la de parejas casadas - la correlación entre fantasías de intimidad con la pareja y fantasías de infidelidad probablemente sea significativa y de mayor magnitud en ambos sexos.

¿Por qué la correlación positiva entre intimidad con la pareja y serle infiel resultó significativa entre las participantes femeninas de este estudio y no entre los participantes masculinos? La correlación significativa en las participantes femeninas probablemente sea una correlación espuria mediada por la actividad sexual con la pareja que estimula el deseo sexual, y este incremento se manifiesta en una mayor frecuencia de fantasías. Las situaciones en las que se reportó tener fantasías sexuales con más frecuencia fueron antes o después de salir con el novio y antes de dormir o después de despertar, sin diferencia significativa entre los participantes de ambos sexos. Al parcializar la varianza de la variable tener fantasías sexuales antes o después de salir con el novio, la correlación entre los contenidos de intimidad con la pareja e infidelidad, estimada por el coeficiente producto-momento de Pearson, dejó de ser significativa en las participantes femeninas $\left(r_{p}=0,06, p=0,37\right.$, cuando la correlación sin parcializar fue $r=0,17, p=0,01$ y la correlación de antes o después de salir con el novio fue alta con el contenido romántico: $r=0,503, p<0,001$ ).

Se suele reportar que las fantasías románticas son más frecuentes en mujeres que en hombres; no obstante, la diferencia es pequeña y resulta el contenido más frecuente en ambos sexos (Holly, 2005; Wilson, 2010). Desde la perspectiva evolutiva, se justifica en cuanto la estrategia reproductiva de la mujer es más selectiva que la del hombre, y la estrategia del hombre es más oportunista que la de la mujer. La mujer busca una pareja que la apoye en la crianza, y el hombre tener el máximo de oportunidades de reproducción, incluso sin implicarse en la crianza (Buss, 2007; Schmitt et al., 2012). Entre los estudiantes de psicología de la presente muestra no probabilística, la diferencia entre ambos sexos no fue significativa. Tampoco en el estudio cualitativo de Moral (2010) hubo diferencia significativa en la frecuencia con la que se contaron fantasías románticas como la fantasía sexual favorita o más frecuente, entre los 66 hombres y las 253 mujeres que dijeron tener fantasías sexuales $\left(\chi^{2}[1, N=319]=3,43, p=0,064\right.$; con la corrección de Yates: $\left.\chi^{2}[1, N=319]=2,81, p=0,088\right)$. Si esta frecuencia de fantasías románticas estadísticamente equivalente entre ambos sexos es un rasgo de la población de estudiantes de psicología, podría atribuirse a una mayor feminidad en el rol de género entre los hombres que eligen estudiar esta carrera, como sugiere el estudio de Taylor (2011). Se podría indagar sobre 
esta hipótesis, aplicando una escala de rol de género al estudiar la diferencia en las medias de frecuencia de fantasías románticas entre ambos sexos en distintas poblaciones.

Se estimaron los deciles en respuesta al cuarto objetivo tanto en la muestra conjunta, como en las muestras de ambos sexos, en razón a la mayor frecuencia de contenidos y situaciones entre los participantes masculinos que entre las participantes femeninas, así como por el tamaño del efecto mediano del sexo sobre la frecuencia del reporte de contenidos y situaciones. Una puntuación de 1 en las escalas de contenidos o situaciones indicaría claramente una inhibición de las fantasías sexuales entre los 400 participantes de ambos sexos, lo cual podría ser motivado por depresión, aversión al sexo o represión sexual (Leiblum, 2014). Cuestión muy relevante en un futuro profesional que se puede dedicar al cuidado de la salud sexual, ya que esto podría sesgar su juicio profesional. Puntuaciones mayores o iguales que 1,9 en la escala de contenidos y 2,5 en la escala de situaciones entre las 200 participantes femeninas, y de 2,5 en la escala de contenidos y 3 en la escala de situaciones entre los 200 participantes masculinos, indicarían de forma clara una fantasía sexual destacadamente activa. Aquí sería relevante evaluar el contexto de actividad sexual del joven y rasgos de personalidad, tales como búsqueda de sensaciones sexuales y compulsividad sexual, los cuales también podrían comprometer el juicio y la ética clínica (Leiblum, 2014; Williams et al., 2009).

Como limitación del estudio debe señalarse el empleo de una muestra no probabilística de estudiantes universitarios de una facultad pública de psicología del noreste de México, por tanto las conclusiones de este estudio deben manejarse como hipótesis o expectativas dentro de esta población y datos de comparación con otras poblaciones. Se empleó un muestreo por cuotas de sexo no proporcionales a las cuotas poblacionales con una sobrerrepresentación de hombres. No obstante, el objetivo era lograr dos muestras grandes y equivalentes en participantes de cada sexo a fin de realizar comparaciones de tendencia central. Como fortalezas se tiene que la variabilidad en edad y semestre, así como el método de selección de los participantes hacen que ambas muestras sean bastante representativas de cada sexo dentro de la población.

En conclusión, en la presente muestra no probabilística de 200 estudiantes femeninas y 200 estudiantes masculinos de una facultad pública de psicología del noreste de México, las distribuciones de la mayoría de los ítems de contenido, de las dos escalas y los dos factores de contenido del cFss mostraron sesgo hacia las frecuencias bajas y no se ajustaron a un modelo de curva normal. El promedio en fantasías heterosexuales de exploración fue significativamente mayor que el de fantasías con personas del mismo sexo, sexo sin coito vaginal y pasividad, siendo esta diferencia mayor en hombres que en mujeres, lo cual es concordante con la ideología heterosexista dominante y los valores homofóbicos más centrados en la sexualidad masculina dentro de la cultura occidental a la que pertenecen los participantes. Los promedios de frecuencia en los contenidos y situaciones fueron mayores en los hombres que en las mujeres, salvo en los contenidos de sexo con personas del mismo sexo, con personas maduras y con parejas concurrentes, así como en las situaciones antes o después de salir con la pareja, y antes o después de dormir. Debido a la falta de normalidad, las puntuaciones en las dos escalas y los dos factores de contenido deben ser baremadas por cuantiles diferenciales para ambos sexos.

A raíz de los presentes datos se formuló como hipótesis la existencia de un sesgo deflactor del reporte de la frecuencia de contenidos y situaciones mediado por la deseabilidad social. Guiados por esta hipótesis, se sugiere estudiar la relación del cuestionario CFSs con el manejo de la impresión y el autoengaño (Ackerman y Wolman, 2007). Asimismo, se podría evaluar la actitud implícita hacia la sexualidad (Cárdenas y Barrientos, 2008), y ver su relación con el cuestionario CFSs. Si existe tal sesgo, estas relaciones serán significativas. En caso de un tamaño de efecto moderado, requeriría su control; pero no así, si el tamaño de efecto es trivial o pequeño. Se podría complementar este cuestionario con el desarrollo de una escala breve de fantasías románticas. Finalmente, se sugiere el estudio y el empleo del cuestionario a fin de evaluar posibles sesgos que puedan afectar al juicio clínico en psicólogos que se capaciten en terapia y educación sexual. En estos estudios se sugiere el uso de un muestreo probabilístico.

\section{Referencias}

Ackerman, P. L. y Wolman, S. D. (2007). Determinants and validity of self-estimates of abilities and self-concept measures. Journal of Experimental Psychology: Applied, 13(2), 57-78.

Ahrold, T. K., Farmer, M., Trapnell, P. D. y Meston, C. M. (2011). The relationship among sexual attitudes, sexual fantasy, and religiosity. Archives of Sexual Behavior, 40, 619-630. doi:10.1007/s10508-010-9621-4 
American Psychological Association (2002). Ethical principles of psychologists and code of conduct. American Psychologist, 57, 1060-1073. doi:10.1037/ 0003-066X.57.12.1060

Bartels, R. M. y Gannon, T. A. (2011). Understanding the sexual fantasies of sex offenders and their correlates. Aggression and Violent Behavior, 16, 551-561. doi: 10.1016/j.avb.2011.08.002

Binter, J., Leongómez, J. D., Moyano, N., Valentova, J., Jouza, L. y Klapilova, K. (2012). Sex differences in the incidence of sexual fantasies focused on evolutionary relevant objects. Anthropologie, 50(1), 83-93.

Birnbaum, G. E. (2007). Beyond the borders of reality: Attachment orientations and sexual fantasies. Personal Relationships, 14, 321-342. doi:10.1111 /j.1475-6811.2007.00157

Brotto, L. A., Jane, S. T., Woo, J. S. T. y Gorzalka, B. B. (2012). Differences in sexual guilt and desire in East Asian and Euro-Canadian men. Journal of Sex Research, 49, 594602. doi:10.1080/00224499.2011.618956

Buss, D. (2007). The evolution of human mating. Acta Psychologica Sinica, 39, 502-512.

Cárdenas, M. y Barrientos, J. (2008). Actitudes explícitas e implícitas hacia los hombres homosexuales en una muestra de estudiantes universitarios en Chile. Psykhe, 17(2), 17-25. doi:10.4067/S0718-22282008000200002

Carlstedt, M., Bood, S. A. y Norlander, T. (2011). The affective personality and its relation to sexual fantasies in regard to the Wilson Sex Fantasy Questionnaire. Psychology, 8, 792-796. doi:10.4236/psych.2011.28121

Critelli, J. y Bivona, J. (2008). Women's erotic rape fantasies: an evaluation of theory and research. The Journal of Sex Research, 45, 57-70. doi: 10.1080/00224490701808191

Darré, S. (2005). Políticas de género y discurso pedagógico. La educación sexual en el Uruguay del siglo xx. Montevideo: Trilce.

De-Craene, V. y Loopmans, M. (2014). Students studying student sexuality: Methodological and ethical implications. Global Studies of Childhood, 4, 276-285. doi:10.2304/gsch.2014.4.4.276

Fisher, S. y Greenberg, R. P. (1996). Freud scientifically reappraised: Testing the theories and therapy. Oxford: John Wiley \& Sons.

Foster, E. K. (2004). Research on gossip: Taxonomy, methods, and future directions. Review of General Psychology, 8, 78-99. doi:10.1037/1089-2680.8.2.78

Goldey, K. L., Avery, L. R. y Van-Anders, S. M. (2014). Sexual fantasies and gender/sex: A multimethod approach with quantitative content analysis and hormonal res- ponses. Journal of Sex Research, 51, 917-931. doi: 10. 1080/00224499.2013.798611

Herek, G. M. y McLemore, K. A. (2013). Sexual prejudice. Annual Review of Psychology, 64, 309-333. doi:10.1146/ annurev-psych-113011-143826

Holly, M. J. (2005). A comparative study of attitudes of sexuality, sexual health knowledge, and sexual practices of young women in Mexico, Canada and the United States. UW-L Journal of Undergraduate Research, 8(1), $1-14$.

Huberman, J. S., Suschinsky, K. D., Lalumière, M. L. y Chivers, M. L. (2013). Relationship between impression management and three measures of women's self-reported sexual arousal. Canadian Journal of Behavioural Science, 45, 259-273. doi:10.1037/a0033397

Kaplan, R. M. y Saccuzzo, D. P. (2009). Psychological testing: Principles, applications, and issues ( $\left.7^{\mathrm{a}} \mathrm{ed}.\right)$. Belmont: Wadsworth.

Leiblum, S. (2014). Sex therapy today, current issues and future perspectives. En S. Leiblum (Ed.), Principles and practice of sex therapy ( $4^{\mathrm{a}}$ ed., pp. 3-22). New York: Guilford Press.

Maciel, M. y Laganà, L. (2014). Older women's sexual desire problems: Biopsychosocial factors impacting them and barriers to their clinical assessment. BioMed Research International, 2014 (article ID 107217), 1-9. doi:10.1155/2014/107217

Moral, J. (2010). Fantasías sexuales en estudiantes universitarios mexicanos. Revista Interamericana de Psicología, 44, 246-255.

Moral, J. (2015). Estructura factorial y consistencia interna del cuestionario de fantasías y situaciones sexuales en estudiantes universitarios mexicanos. Revista Ciencia UAT, 10(1), 17-31.

Moral, J. y Ortega, M. E. (2009). Representación social de la sexualidad y actitudes en estudiantes universitarios mexicanos. Revista de Psicología Social, 24, 65-79. doi:10.1174/021347409786922998

Morgade, G. (2006). Sexualidad y prevención: discursos sexistas y heteronormativos en la escuela media. Revista del IICE, 24(1), 27-33.

Petersen, J. L. y Hyde, J. S. (2010). A meta-analytic review of research on gender differences in sexuality, 1993 2007. Psychological Bulletin, 136, 21-38. doi:10.1037/ a0017504

Rathus, S. A., Nevid, J. S., Fichner-Rathus, L., Herold, E. S. y McKenzie, S. W. (2005). Human sexuality in a world of diversity ( $2^{\mathrm{a}}$ ed.). Upper Saddle River, NJ: Pearson. Education. 
Remafedi, G., Resnick, M., Blum, R. y Harris, L. (1992). Demography of sexual orientation in adolescents. Pediatrics, 89, 714-721.

Schmitt, D. P. Jonason, P. K. Byerley, G. J., Flores, S. D., Brittany E. Illbeck, B. E., O’Leary, K. N. y Qudrat, A. (2012). A reexamination of sex differences in sexuality: New studies reveal old truths. Current Directions in Psychological Science, 21, 135-139. doi:10.1177/0963721412436808

Seifert, K. M., Boulas, J. C., Huss, M. y Scalora, M. (marzo, 2011). Response bias on self-report measures of sexual fantasies. Trabajo presentado en el 4th International Congress of Psychology and Law. Organizado por la American Psychology-Law Society. Miami, FL. Resumen recuperado de: http://citation.allacademic.com/ meta/p483364_index.html

Sierra, J. C., Ortega, V. y Zubeidat, I. (2006). Confirmatory factor analysis of a Spanish version of the Sex Fantasy Questionnaire: Assessing gender differences. Journal of Sex and Marital Therapy, 32, 137-159. doi:10.1080/00926230500442318

Sociedad Mexicana de Psicología (2007). Código ético del psicólogo ( $4^{\mathrm{a}}$ ed.). Ciudad de México: Editorial Trillas.

Sylva, D., Safron, A., Rosenthal, A. M., Reber, P. J., Parrish, T. B. y Bailey, J. M. (2013). Neural correlates of sexual arousal in heterosexual and homosexual women and men. Hormones and Behavior, 64, 673-684. doi: 10.1016/j.yhbeh.2013.08.003

Taylor, C. (2011). Sexually dimorphic traits and masculinity/femininity: Using speed-dating to explore human attraction (Honours thesis, School of Psychology,
The University of Queensland, Brisbane, Australia). Recuperada de: http://espace.library.uq.edu.au/view/ UQ:276396

Tourangeau, R. y Yan, T. (2007). Sensitive questions in surveys. Psychological Bulletin, 133, 859-883. doi:10.1037/ 0033-2909.133.5.859

Tuval-Mashiach, R., Walsh, S., Harel, S. y Shulman, S. (2008). Romantic fantasies, cross-gender friendships, and romantic experiences in adolescence. Journal of Adolescent Research, 23, 471-487. doi:10.1177/ 0743558407311332

Vázquez, V. y Chávez, M. (2008). Género, sexualidad y poder. El chisme en la vida estudiantil de la Universidad Autónoma de Chapingo, México. Estudios sobre las Culturas Contemporáneas, 14(27), 77-112.

Vrangalova, Z. y Savin-Williams, R. C. (2010). Correlates of same-sex sexuality in heterosexually identified young adults. Journal of Sex Research, 47, 92-102. doi: 10.1080/00224490902954307.

Wilson, G. D. (2010). Measurement of sex fantasy. Sexual and Relationship Therapy, 25, 57-67. doi:10.1080/14681990903550134

Williams, K. M., Cooper, B. S., Howell, T. M., Yuille, J. C. y Paulhus, D. L. (2009). Inferring sexually deviant behavior from corresponding fantasies. Criminal Justice and Behavior, 36, 198-222. doi:10.1177/0093854808327277

Zumbo, B. D., Gadermann, A. M. y Zeisser, C. (2007). Ordinal versions of coefficients alpha and theta for Likert rating scales. Journal of Modern Applied Statistical Methods, 6, 21-29. 


\section{Apéndice A}

\section{Cuestionario de Fantasías Sexuales y Situaciones (CFSS)}

Indique con qué frecuencia tiene cada una de las siguientes fantasías sexuales, y con qué frecuencia tiene fantasías sexuales en cada una de las siguientes situaciones.

\begin{tabular}{|c|c|c|c|c|c|}
\hline \multirow{2}{*}{ FRECUENCIA } & 1 & 2 & 3 & 4 & 5 \\
\cline { 2 - 6 } & Nunca & Muy rara vez & A veces & Con frecuencia & Con mucha frecuencia \\
\hline
\end{tabular}

\begin{tabular}{|c|c|c|c|c|c|c|}
\hline & Contenidos & \multicolumn{5}{|c|}{ Frecuencia } \\
\hline $\mathrm{C} 1$ & ser masturbado/a, tener sexo oral o anal & 1 & 2 & 3 & 4 & 5 \\
\hline c2 & $\begin{array}{l}\text { Tener relaciones sexuales en un avión, manejando o en situaciones de } \\
\text { riesgo }\end{array}$ & 1 & 2 & 3 & 4 & 5 \\
\hline c3 & Tener relaciones bisexuales & 1 & 2 & 3 & 4 & 5 \\
\hline $\mathrm{C} 4$ & Tener relaciones homosexuales & 1 & 2 & 3 & 4 & 5 \\
\hline c5 & ser infiel a mi pareja & 1 & 2 & 3 & 4 & 5 \\
\hline c6 & Participar en una orgía heterosexual & 1 & 2 & 3 & 4 & 5 \\
\hline c7 & Hacer un intercambio de parejas & 1 & 2 & 3 & 4 & 5 \\
\hline c8 & Hacerlo con un hombre famoso o una mujer famosa & 1 & 2 & 3 & 4 & 5 \\
\hline C9 & ser el amante o hacerlo con una persona casada & 1 & 2 & 3 & 4 & 5 \\
\hline $\mathrm{C} 10$ & Hacerlo con una persona madura & 1 & 2 & 3 & 4 & 5 \\
\hline $\mathrm{c} 11$ & Tener relaciones sexuales con el/la novio/a de mi amigo/a & 1 & 2 & 3 & 4 & 5 \\
\hline $\mathrm{C} 12$ & Tener relaciones con alguien mucho más joven que yo & 1 & 2 & 3 & 4 & 5 \\
\hline $\mathrm{C} 13$ & Que me aten, me golpeen, me sometan o abusen de mí & 1 & 2 & 3 & 4 & 5 \\
\hline \multirow[t]{2}{*}{ c14 } & Observar a otros teniendo relaciones sexuales & 1 & 2 & 3 & 4 & 5 \\
\hline & situaciones & \multicolumn{5}{|c|}{ Frecuencia } \\
\hline s1 & Cuando estudio o estoy aburrido & 1 & 2 & 3 & 4 & 5 \\
\hline s2 & Antes de dormir o al despertar & 1 & 2 & 3 & 4 & 5 \\
\hline s3 & Viendo la televisión & 1 & 2 & 3 & 4 & 5 \\
\hline s4 & Cuando llevo muchos días sin masturbarme o tener actividad sexual & 1 & 2 & 3 & 4 & 5 \\
\hline s5 & Después o antes de salir con mi pareja & 1 & 2 & 3 & 4 & 5 \\
\hline s6 & $\begin{array}{l}\text { Cuando deseo a alguien que no me atrevo a conquistar o no me es } \\
\text { accesible }\end{array}$ & 1 & 2 & 3 & 4 & 5 \\
\hline s7 & Cuando deseo a alguien que me rechaza & 1 & 2 & 3 & 4 & 5 \\
\hline s8 & Cuando estoy en el chisme o se platica de cosas sexuales & 1 & 2 & 3 & 4 & 5 \\
\hline s9 & Cuando estoy en la ducha o el baño & 1 & 2 & 3 & 4 & 5 \\
\hline s10 & Después de tener actividad sexual con mi pareja & 1 & 2 & 3 & 4 & 5 \\
\hline
\end{tabular}

